
VIVIANN MOANA WILMOT / MIRJAM ELOMDA /

CÉCILE STEHRENBERGER Und URS LINDNER

im Gespräch mit NAOMIE GRAMLICH und JANA MANGOLD

\section{ERFURT DEKOLONISIEREN}

1 Vgl. dazu die Erklärung des bundesweiten Netzwerks zur Dekolonisierung der Erinnerungskultur, 23.11.2018, isdonline. de/100-jahre-nach-dem-ende-desdeutschen-kolonialreichs-erklaerungdes-bundesweiten-netzwerks-zurdekolonisierung-der-erinnerungskultur| (18.2.2020).

2 Decolonize Erfurt hat im Jahr 2019 mehr als zehn Stadtrundgänge angeboten. Die Ausstellung «Kolonialismus in Erfurt, 1503 bis heute» war 2019 im Haus Dacheröden, im Thüringer Landtag sowie im Foyer der Erfurter Universitätsbibliothek zu sehen. Darin war auch Material aus der von der ISD konzipierten Ausstellung «Homestory Deutschland. Schwarze Biografien in Geschichte und Gegenwart» zu sehen.
Städtische Räume in Deutschland zeigen noch heute zahlreiche Spuren und Einschreibungen aus der Zeit der deutschen Kolonialgeschichte. Das zivilgesellschaftliche Netzwerk zur Dekolonisierung der Erinnerungskultur ${ }^{1}$ unternimmt im Zusammenschluss mit Vereinen wie der Initiative Schwarze Menschen in Deutschland (ISD) seit einigen Jahren die Aufarbeitung des kolonialen Erbes in deutschen Städten. Teil des Netzwerks ist auch die Initiative Decolonize Erfurt, die sich mit lokalen Ausprägungen und Besonderheiten der Kolonialgeschichte und deren Fortwirken in der Gegenwart der Stadt beschäftigt. Mitglieder von Decolonize Erfurt haben im Wintersemester 2018/19 ein fächerübergreifendes Seminar an der Universität Erfurt initiiert, in dem ein dekolonialer Stadtrundgang sowie die Ausstellung «Kolonialismus in Erfurt, 1503 bis heute» entwickelt wurden. ${ }^{2}$

Wir trafen Mirjam Elomda von der ISD Thüringen, Cécile Stehrenberger und Urs Lindner von Decolonize Erfurt sowie Viviann Moana Wilmot, die in beiden Gruppen mitgewirkt hat, und sprachen mit ihnen über Grenzen und Potenziale des dekolonialen Stadtrundgangs für antirassistische Arbeit, Zeitlichkeiten und Sichtbarmachung von Kolonialität und über migrantische Erfahrungen im ostdeutschen Kontext. Das Gespräch führten wir am selben Tag, an dem die Akteur_innen auch zu einer öffentlichen Podiumsdiskussion mit der Erfurter Stadtverwaltung eingeladen waren. Die Langversion des Gesprächs findet sich unter www.zfmedienwissenschaft.de/online.

Mirjam Elomda Ein Hinweis zu Beginn: Ich bitte euch, dass im Rahmen des Gesprächs nur Eigenbezeichnungen verwendet werden, und wenn rassistische Begrifflichkeiten genannt werden, dass dies zumindest vorher angekündigt wird. 
Jana Mangold Unser Gespräch würde ich gerne mit dem gemeinsamen Blick auf ein Foto beginnen, das ich gestern in der Andreasstraße in Erfurt aufgenommen habe. Aufgefallen ist mir das Abgebildete vor ungefähr zwei Wochen, also zwei Wochen nach meinem letzten Decolonize-Erfurt-Stadtrundgang. Ich möchte euch bitten, etwas dazu zu sagen, wie das Abgebildete auf euch wirkt.

Naomie Gramlich Achtung: Es handelt sich um eine rassifizierende Darstellung.

M.E. Mir ist es nie aufgefallen. Es wirkt so frisch restauriert.

Urs Lindner Frisch erfunden!

M.E. Da hat sich jemand noch mal Mühe gemacht.

J.M. Der Straßenzug, in dem es sich befindet, wurde über mehrere Jahre saniert.

Viviann Moana Wilmot Ich meine, das wäre früher mal eine Bar gewesen, mit dem Namen $<$ Zum $M>{ }^{3}$ oder so ähnlich. Ich habe meine BA-Arbeit über Kolonialität am Beispiel des Stadtfests in Eisenberg geschrieben. ${ }^{4}$ Im Zuge dessen habe ich zum M-Wort in Thüringen recherchiert und herausgefunden, wie präsent es tatsächlich im Erfurter Stadtbild war und ist. Es gibt auch hier eine Apotheke, die so heißt, die - um Dienstbarkeit zu symbolisieren - einen knienden Schwarzen Menschen als Figur im Schaufenster ausstellt. Es gibt eine Gasse, die den M-Namen trägt. Und es gibt immer noch das $<$ Haus zum M-Kopf $>$ in der Johannesstraße ... U.L. Es gibt zehn Häuser mit M-Namen in Erfurt.

V.M.W. ... und es gab verschiedene Gaststätten, Kneipen. Und ich glaube, dass diese klischeebehaftete Darstellung eben auch an einem Haus angebracht ist, wo früher so eine Kneipe war - also dem Namen der Stätte entsprechend. Es wurde nichts umbenannt, sondern die Häuser, die es heute nicht mehr gibt, sind einfach verfallen.

M.E. Interessant finde ich, dass dir diese Darstellung im Vorfeld des Gesprächs aufgefallen ist, weil es zeigt, wie sensibel man mit dem Thema umgehen kann. Die genaue Ausprägung der Darstellung reproduziert rassistische Darstellungen von Schwarzen Menschen. Das ist einfach wieder diese typische Darstellung mit dicken Lippen und diesen Ringen in den Ohren. Das sind die ersten Gedanken, die ich dazu habe: Zum einen die Sensibilität hinsichtlich kolonialer Spuren im Stadtraum und zum anderen, dass diese Darstellung nicht zeitgemäß ist, zumindest nicht, sie unkommentiert stehen zu lassen.

U.L. Ich würde sagen, die Darstellung ist ganz klar rassistisch, weil Schwarze Menschen über u. a. den Ohrring - hier kann man es auch sehen - mit <Wildheit> und $\langle\text { Kannibalismus }\rangle^{\mathbf{5}}$ assoziiert werden. Das ist um I900 das, was der Ohrring in den Darstellungen symbolisieren soll. Nun wäre es interessant zu wissen, von wann das hier ist. Ich bin mir ziemlich sicher, dass es aus dem I9. oder 20. Jahrhundert ist. Ich kann mir nicht vorstellen, dass es älter ist - nach allem, was ich weiß. Es ist allerdings immer schwierig, genaue Daten herauszufinden.

Cécile Stehrenberger Wie schon gesagt: Es ist erst vor Kurzem restauriert worden. Das heißt, wenn wir fragen: Von wann ist es?, müssen wir sagen: von
3 Rassistische Begriffe und Fremdbezeichnungen werden hier nicht wiedergegeben, sondern mit dem Anfangsbuchstaben abgekürzt. Stattdessen werden Selbstbezeichnungen wie ‘Schwarz (mit großem Anfangsbuchstaben) oder (People of Color (PoC), gewählt. «Weiß) (kleinund kursiv geschrieben) steht für einen analytischen Begriff, der die rassistische Konstruktion der Moderne markiert. Für eine umfassende Beschäftigung mit rassistischen Sprachhandlungen siehe Susan Arndt, Nadja Ofuatey-Alazard: Wie Rassismus aus Wörtern spricht: (K)erben des Kolonialismus im Wissensarchiv deutsche Sprache. Ein kritisches Nachschlagewerk, Münster 2011.

4 Viviann Moana Wilmot: Das M-Wort als Ausdruck von Kolonialität - anhand eines aktuellen Beispiels aus Thüringen, in: Wissen schafft Demokratie, Bd. 7, 2020 (Schriftenreihe des Instituts für Demokratie und Zivilgesellschaft), in Vorbereitung. Die Stadt Eisenberg, ca. $80 \mathrm{~km}$ östlich von Erfurt gelegen, hat 2019 ihr Stadtfest in "M-Fest" umbenannt. Der neue Name wird auf eine regionale Sage mit der Figur eines $M$ sowie auf Stadtwappen und Stadtbrunnen, die eine stereotype, rassistische M-Figur zeigen, zurückgeführt. Die ISD hat die Stadt in einem offenen Brief zum Überdenken und Gegensteuern aufgefordert. Siehe: Thembie Wolf: Eisenberg feiert bald ein «Mohrenfest». Konrad will das verhindern, in: bento, zuletzt aktualisiert 27.5.2019, hdl.handle. net $/ 11346 / N 8 C Q$ (18.2.2020). 5 (Kannibalismus) und (Wildheit) sind kolonialistische, weiße Erfindungen zur Herabsetzung von BIPoCs (Black/Indigenous/People of Color) und dienen einer Rechtfertigungsrhetorik europäischer Expansionen. Vgl. «Kannibalismus», in: Arndt, Ofuatey-Alazard: Wie Rassismus aus Wörtern spricht, $691 \mathrm{f}$. 
6 Homestory Deutschland. Schwarze Biografien in Geschichte und Gegenwart, siehe www.homestory-deutschland.de. 7 Seine Disputation mit dem Titel «Über die Rechtsstellung der $M$ in Europa" hielt er 1729, siehe www.homestory-deutschland.del biografien/anton-wilhelm-amo/detail. html (18.2.2020). heute! Wenn es nicht kommentiert ist, ist es in seinem Nicht-kommentiertWerden von heute. Das ist einer der wichtigsten Aspekte daran - und natürlich zu wissen, dass es sich um kein isoliertes Phänomen handelt, sondern überall in der Stadt anzutreffen ist.

N.G. Auch wenn es sich bei diesem Bild eben nicht um ein Individuum handelt, sondern um eine typisierende, rassifizierende Darstellung eines Schwarzen Menschen, verweist der Begriff $\langle\mathbf{M}\rangle$ auch auf die Geschichte der Bediensteten an europäischen Adelshöfen seit dem 18. Jahrhundert. Bist du, Viviann, im Zuge deiner Recherche für deine BA-Arbeit auch auf afrikanische Präsenz in Erfurt bzw. in Thüringen gestoßen?

V.M.W. Zum Glück hat die Aufarbeitung Schwarzer Präsenz in Deutschland bereits begonnen. So z. B. mit der Ausstellung «Homestory Deutschland» ${ }^{\mathbf{6}}$, die die ISD zusammengestellt hat und die Schwarze Präsenz über drei Jahrhunderte abbildet. Ein prominentes Beispiel für Schwarze Präsenz in Thüringen ist Anton Wilhelm Amo, der als $\langle$ Hof-M〉 am Schloss von BraunschweigWolfenbüttel gedient hat. Später konnte er eine Ausbildung genießen und letztendlich Ende I 720 in den Rechtswissenschaften in Halle promovieren. ${ }^{7}$ Seine letzte Station in Deutschland war Jena, wo er an der Universität gelehrt hat. Im Zuge von rassistischen Schmähkampagnen um I 740 musste er das Land verlassen. Heute erinnert in Jena eine unscheinbare Plakette an seinem letzten Wohnhaus an ihn.

Die Aufarbeitung dieser Geschichten ist eine schwierige Aufgabe. Oft kennen wir nicht einmal die Namen der Betroffenen, da sie als versklavte Menschen nach Deutschland bzw. in die deutschen Vorgänger-Staaten kamen und als sogenannte $\langle$ Hof- $\mathrm{M}\rangle$ an deutschen Adelshöfen dienen mussten. Diese Tatsache zeigt auch nochmal, wie stark das M-Wort verknüpft ist mit der Tradition der Ausbeutung Schwarzer Menschen in Deutschland.

Ich denke aber nicht, dass sich von der Verbreitung des M-Wortes in Erfurts Stadtbild auf Schwarze Präsenz in Thüringen schließen lässt. Vielmehr zeugen Darstellungen wie auf dem Foto von einer stereotypisierten Vorstellung von Schwarzsein - die wenig mit tatsächlichem Kontakt zu tun hat.

N.G. Es gibt ja auch nie Namensschilder dazu. Sie sind entindividualisiert. Es gibt keine Menschen dahinter.

U.L. Genauso wie bei dem $M$ da in Eisenberg, bei dem Stadtmaskottchen. Der hat ja auch keinen Namen. Das ist ja kein Individuum, sondern ein Typus, der dargestellt wird.

C.S. Ich würde sogar noch weiter gehen und sagen, dass das eine Tradition der Behandlung ist, die enthumanisiert.

J.M. Auf diese Problematiken macht ihr bei euren Stadtrundgängen aufmerksam. Für mich sind die Rundgänge ein niedrigschwelliges Angebot, mich mit 
den Fragen der Kolonialgeschichte und dem Wirken des Kolonialismus vor meiner Haustür auseinanderzusetzen. Ich bin aber auch eine Person mit einem (kulturwissenschaftlichen) Studium und somit aus einer privilegierten Schicht der Gesellschaft. Wer ist euer Publikum bei den Stadtrundgängen? Wen wollt ihr erreichen? Oder geht es euch selbst um eine Auseinandersetzung mit der Kolonialgeschichte in der Stadt, in der ihr lebt?

U.L. Ich habe durch die Beschäftigung mit dem Kolonialismus in Erfurt sehr viel über diese Stadt gelernt. Ich bin auch zu der Überzeugung gekommen, dass der Stadtrundgang ein gutes politisches Format ist. Weil die Leute - wenn der Stadtrundgang gut läuft und sie sich darauf einlassen - lernen, die Stadt, in der sie leben, mit anderen Augen zu sehen. Das bringt viel mehr, als etwa eine Veranstaltung mit einem Vortrag zu organisieren. Wenn es um Subjektivität geht - und Subjektivität hat ja auch etwas mit dem räumlichen Empfinden und Erleben zu tun -, kann man mit dem Stadtrundgang wirklich einiges erreichen. Für unsere Stadtrundgänge kooperieren wir mit verschiedenen Einrichtungen und Vereinen, wie z.B. mit der Fachhochschule, der AWO oder der Gambia-Gruppe aus Erfurt. Entsprechend den jeweiligen Interessen stellen wir die einzelnen Stationen zusammen. Dabei ist immer das Ziel, allgemein verständlich und niedrigschwellig zu bleiben. Der Stadtrundgang soll nicht etwas sein, dem man nur mit einer akademischen Ausbildung folgen kann.

C.S. Stimmt, wir versuchen die Rundgänge zugänglich zu gestalten. Trotzdem sind unsere Rundgänge nicht so konzipiert, dass sie leicht verdauliche Unterhaltung bieten. Ich sage das, weil es in Erfurt einige Rundgänge gibt, die dies tun, mit vielen kurios amüsanten Anekdoten, Kostümierung oder Verpflegung. Unser Stadtrundgang kann und soll dagegen in einem gewissen Maße irritieren und verunsichern. Für mich ist es immer wichtig, eine Aktualität der Themen zu zeigen, wofür wir die Verbindung von Ausstellung, Website und Rundgang nutzen. ${ }^{8}$

N.G. Was mir bei den Reaktionen auf antirassistische und post- bzw. dekoloniale Arbeit immer wieder auffällt, ist, dass nicht so sehr Kolonialgeschichte als historischer Fakt bestritten wird, sondern ein komplexes Verständnis von Kolonialität und Rassismus fehlt. Als ich mal einem Botaniker gegenüber erwähnt habe, dass nicht nur die Bestände botanischer Gärten einen kolonialen Ursprung haben, sondern die Praktiken des Sammelns, Klassifizierens und Umbenennens von Pflanzen an sich bereits wissenschaftlicher 
8 Website von Decolonize Erfurt: decolonizeerfurt.wordpress.com/. 9 Siehe auch den Beitrag "Baumwolle: Gefüge mit Gewalt» von Ulrike Bergermann in diesem Heft, 122-129.

10 Das beanspruchte Gebiet «Deutsch-Ostafrikas» umfasste Tansania, Burundi, Ruanda und einen kleinen Teil Mosambiks.
Ausdruck kolonialer Bestrebungen seien, reagierte der mit der Aussage: «Man muss es nicht noch kolonialer machen, als es ohnehin schon ist.» Dieser Satz ist natürlich ein Paradox und versucht, die Adressat_in des Problems zur Verursacher_in zu machen. Der Satz zeigt aber auch, dass es zwar ein ungefähres Wissen über Kolonialismus gibt, aber kein angemessenes Verständnis darüber, was Kolonialität auf begrifflicher, epistemologischer, wissenschaftlicher, ja insbesondere ethisch-politischer Ebene eigentlich heißen könnte bzw. heiBen müsste. Um es mit Ann Laura Stoler zu sagen, Kolonialismus wird nicht einfach vergessen, vielmehr herrscht eine Art Aphasie, eine Sprachlosigkeit. Wie würdet ihr aus eurer Erfahrung heraus diese Art des (Nicht-)Umgangs beschreiben? Was bleibt alles unsichtbar bzw. unsagbar, wenn ihr einzelne Stationen der Stadt ablauft?

U.L. Wir haben bei den Stadtrundgängen immer darauf geachtet, eine Mischung aus Stationen zu haben, die sowohl das Allgemeine des Kolonialismus als auch das Erfurt-Spezifische thematisieren, das Sichtbare wie das Unsichtbare. Deswegen ist es mir z.B. immer wichtig, zum Burenhaus zu gehen. Es wurde I90I/o2 erbaut, erinnert an den zweiten Burenkrieg (I 899-1902) und ehrt an seiner Fassade die ideologischen und politischen Begründer der südafrikanischen Apartheid. Das Burenhaus ist das einzige (erhaltene) dieser Art in ganz Deutschland und zugleich das eindrücklichste sichtbare koloniale Monument in Erfurt. Dann gibt es Stationen wie die «Kolonialität des Smartphones», wo man das Handy hochhalten und sagen kann: «Hier drin steckt die Fortsetzung der Kolonialgeschichte». Aber das hat keine spezifische Sichtbarkeit in Bezug auf Erfurt. Und schließlich gibt es Mittelstationen, wie z.B. das Haus Dacheröden. Im I9. Jahrhundert war es die Firmenzentrale der Firma Johann Anton Lucius, die mit Baumwolle gehandelt hat. Baumwolle war eine der wichtigsten kolonialen Waren. ${ }^{9}$ Ende des I9. Jahrhunderts waren die Lucius die reichste Familie Erfurts. Und sie hatten Anfang des 20. Jahrhunderts auch noch eine Baumwollplantage im damaligen DeutschOstafrika. ${ }^{10}$ Hier haben wir Wertschöpfungsketten, die nicht direkt sichtbar sind, und das immer noch sehr sichtbare Haus der ehemaligen Firmenzentrale.

C.S. Um diese Unsichtbarkeit des Kolonialen an den Orten und Dingen, die zu den sichtbarsten und meist gesehenen der Stadt gehören, geht es uns auch an anderen Stationen.

U.L. So ist Erfurt seit dem I9. Jahrhundert als <Blumenstadt $>$ bekannt und wird auch bis heute als solche touristisch vermarktet - insbesondere an Orten wie der Erfurter Gartenausstellung. Selbstverständlich ohne, dass dabei die lokale (!) koloniale Geschichte der Blumen, die überall zu sehen sind, sichtbar gemacht würde: Da ist z. B. das Usambara-Veilchen. Es wurde von einem Berliner Kolonialbeamten, Walter von Saint Paul-Illaire, im heutigen Tansania im Jahr I892 <entdeckt〉. Im Jahr darauf wurde die Pflanze durch einen Hannoveraner Botaniker klassifiziert und nach ihrem <Entdecker> benannt: Saintpaulia ionantha. Die Firma, die damit große Profite gemacht hat, weil sie die Blume in den Weltsamenhandel eingeführt hat, war die Firma Ernst Benary aus Erfurt. 
V.M.W. Ich glaube, wenn man so einen Stadtrundgang macht und die Leute anschließend rausgehen und sagen: «Ah okay, also wir müssen jetzt die Straße oder das Haus umbenennen, dann haben wir dekolonisiert», dann hat man irgendwas falsch gemacht. Also, das ist ja nicht unsere Botschaft. Natürlich ist es wichtig, auch Stationen, wo sich die Kolonialität z. B. in Form einer rassifizierenden Darstellung Schwarzer Menschen quasi greifen lässt, zu haben. Das ist einfach viel anschaulicher. Und macht ja gerade den Unterschied zu irgendwelchen theoretischen Texten oder irgendwelchen Debatten aus, die nur auf einer intellektuellen Ebene stattfinden. Aber es geht ja immer darum, die Kontinuitäten und Verbindungen aufzuzeigen. Wenn wir über das 〈Haus zum M-Kopf〉 sprechen, sprechen wir über Rassismus. Und den werden wir natürlich nicht los, indem wir ein Haus umbenennen. Aber indem wir solche Bezeichnungen problematisieren und Umbenennung fordern, machen wir rassistische Kontinuitäten sichtbar. Und das ist der Auftrag.

M.E. Was mir noch als aktuelles Beispiel einfällt, wenn es um die Kontinuität von Unsichtbarkeiten nicht nur deutscher Kolonialgeschichte, sondern insbesondere auch Schwarzer Menschen und ihrer Bedeutung für die deutsche Geschichte geht: Der Aktivist und Schwarze Deutsche Theodor Wonja Michael, ein Überlebender des NS-Regimes, ist vor Kurzem verstorben. Die Nachrufe allerdings hielten sich in Grenzen und kamen vor allem aus der Schwarzen Community, dabei war er eine wichtige historische Persönlichkeit in Deutschland. ${ }^{11}$ Ohne Themen vermischen zu wollen, zeigt sich an der Frage der Schwarzen Präsenz eine Kontinuität von Privilegierungen und Deprivilegierungen.

J.M. Mich würde noch interessieren, welches Verhältnis es zwischen der ISD und der Decolonize-Gruppe gibt, und auch, wie die unterschiedlichen Akteur_innen zur Idee eines dekolonisierenden Stadtrundgangs stehen? Wie setzen sich die Gruppen zusammen? Welche Gruppe sagt, wir sind Dekolonisierer_innen? Wo liegen die Aufgaben der ISD?

U.L. In der Gruppe Decolonize Erfurt waren auch Aktivist_innen der ISD beteiligt. Zuletzt Mirjam, wenn ich dich dazuzählen darf ...

M.E. Ich zähle mich nicht dazu.

\section{N.G. Warum nicht?}

M.E. Weil ich ehrenamtlich tätig bin und die Kapazitäten nicht habe, alle Angebote, die es gibt, zu bedienen. Meine Priorität ist, nach innen Arbeit zu leisten für die Community, um diese aus sich heraus zu stärken. Und es dient Schwarzen Menschen nicht, wenn ich bei Decolonize Erfurt aktiv bin und ab und zu einen Stadtrundgang anbiete. Es sind andere Themen, die für die Community in Thüringen brennen. Der Stadtrundgang ist ein wichtiges Bildungsangebot, das wir als Lokalgruppe der ISD gerne unterstützen, weil es die Verknüpfung von Kolonialismus und Rassismus, wie er jetzt besteht, aufzeigen kann.
11 Hadija Haruna, Joshua Kwesi Aikins: Ein Leben, das wir weitertragen werden, in: Zeit Online, 22.10.2019, www.zeit.del kultur/2019-10/theodor-wonja-michaelgestorben-nachruf (18.2.2020). 
12 Z. B. mit Walter D. Mignolo: Delinking. The rhetoric of modernity, the logic of coloniality and the grammar of de-coloniality, in: Cultural Studies, Bd. 21, Nr. 2/3, 2007, 449-514.
V.M.W. Du hast recht. Der Rundgang ist sehr wichtig, aber er deckt den Bedarf nicht. Die Schwarze Community hat viel mehr davon, dass du, Mirjam, z. B. die regelmäßigen Treffen organisierst. Bei dem Stadtrundgang geht es um wichtige Themen, aber er dient nicht selbstverständlich dem Empowerment.

M.E. Das ist ein wichtiger Punkt. Darüber hatten wir uns im Vorfeld zu diesem Gespräch ausgetauscht, dass die postcolonial- und decolonize-Arbeit für Schwarze, marginalisierte Personen oder Personen der afrikanischen Diaspora in erster Linie Biografiearbeit ist. Das sind sehr persönliche Themen. Das ist nebeneinanderzudenken, aber nicht unbedingt miteinander. Deshalb ist der Stadtrundgang auch nicht ein Angebot, für den jede_r empfänglich ist.

U.L. Vielleicht folgt daraus, dass der Stadtrundgang sich primär an Weiße richtet und für diese idealerweise ein Medium sein kann, etwas besser zu verstehen, was Rassismus ist und woher der kommt.

C.S. Was Mirjam auch angesprochen hat - das Zeitproblem - zeigt ja, dass es so viele Orte und Notwendigkeiten gibt, antirassistisch aktiv zu sein. Ich möchte an der Stelle auch betonen, dass innerhalb von Decolonize Erfurt dieser Stadtrundgang immer nur einer von mehreren Betätigungsbereichen war.

N.G. Weil ihr gerade von den Tools dekolonialer Arbeit sprecht, würde ich euch gerne zu eurem Verständnis von <dekolonial> fragen. Die ersten Gruppen, die sich in den r980er und r99oer Jahren in Deutschland mit der Kolonialgeschichte in den Städten beschäftigt haben, hießen noch Hamburg Global oder Berlin Postkolonial. Nun gibt es seit einigen Jahren eine größere Aufmerksamkeit für das Schlagwort <dekolonial>, das auch insbesondere mit Theorien aus Südamerika verbunden ist. ${ }^{12}$ Im Unterschied zu postkolonialen Ansätzen, die meinen, dass Kolonialität zwar historisch abgeschlossen, aber noch nicht vorbei ist, verweist Dekolonialität zumindest nach eigenem Selbstverständnis auf ein radikaleres Konzept, das Kolonialität und Modernität viel stärker miteinander verbunden sieht. Meine Frage ist, ob und wenn ja wie ihr euch in eurem Vorgehen von anderen Gruppen wie Berlin Postkolonial unterscheidet?

U.L. Ich kann das Label <dekolonial> aus zwei Gründen total affirmieren: Zum einen hat die Dekolonisierung mit der staatlichen Unabhängigkeit der ehemaligen Kolonien bisher vor allem formal stattgefunden. In einem substanziellen, die Gesellschaften tatsächlich verändernden Sinn ist sie eine politische Aufgabe, die sowohl die ehemaligen Kolonialmächte als auch die Kolonisierten noch zu leisten haben. Zum anderen ist natürlich auch der Bezug auf die Theorieebene wichtig: Die dekoloniale Perspektive interessiert sich auch für die politischökonomischen Verhältnisse im Unterschied zu vielen postkolonialen Ansätzen, die stark, vielleicht zu stark auf Kultur fokussiert sind. Sie ist ja im Prinzip eine Synthese aus postkolonialer Wissens- und Kulturkritik plus marxistisch geprägter Weltsystem- und Dependenztheorie.

C.S. Gleichzeitig gilt, dass Menschen, die unter dem Label <postkolonial〉 aktiv waren, genauso radikal waren und sind. Trotz Kritik an den theoretischen 
Ansätzen verbindet sich mit dem Wort <postkolonial> ein Programm, das weit über das Wiederkäuen von z. B. Homi K. Bhabha hinausgeht. Das gilt für die Aktivist_innen umso mehr. Der Begriff <Postkolonialismus〉 wurde in den letzten Jahrzehnten in so mannigfaltigen Weisen verwendet, was längst über das hinausgegangen ist, was die Menschen, die den Begriff in den I980er Jahren geprägt haben, damit eigentlich vorgehabt haben. Das Signifikante ist daran vielleicht, dass man das an den weiter vom Aktivismus entfernten Orten, also an der Universität, zu wenig sieht.

v.M.W. Die Gruppe Berlin Postkolonial als eine der ersten Initiativen, die in Deutschland dekoloniale Arbeit leisteten, wäre sicherlich sehr unglücklich darüber, wenn sie wegen des Labels als weniger radikal verstanden werden würde. Die deutschlandweiten Gruppen bezeichnen sich zwar unterschiedlich, leisten inhaltlich aber dieselbe Arbeit. Die Erfurter Initiative ist relativ jung und hat sich bewusst <Decolonize > genannt, wohinter ich total stehe, da die lateinamerikanische Theorie den Anspruch verfolgt, mit sozialen Bewegungen zusammenzuarbeiten. Auch wenn durch die theoretische Komplexität der Texte dieser Anspruch nicht unbedingt realisierbar ist. Außerdem finde ich richtig und wichtig, dass bei <Decolonize! > die Aufforderung bereits im Titel steckt. Die englische Bezeichnung dient dazu, die deutsche Kolonialgeschichte in einen größeren Kontext einzuordnen.

N.G. Da es uns ja auch um Formate, Darstellung und Präsentationsformen sowie deren machtkonsolidierende Wirkung geht, derer wir uns auch dank der postkolonialen Theorie bewusst geworden sind, möchte ich in einer suchenden Richtung nachfragen: Ist der Stadtrundgang wirklich - und das ist natürlich provokativ - das richtige Format? Denn - ihr habt es schon angesprochen - Stadtrundgänge stehen in einer Tradition der Unterhaltung und des show and tell: Man hält laminierte Bilder hoch und die Leute nicken ganz informiert. Dasselbe trifft auf diese <denkwürdige> Einrichtung des Museums und ihr Format der Ausstellung zu. Das wird ja auch innerhalb der Restitutionsdebatte diskutiert, ob das museale Ausstellen überhaupt der richtige Umgang mit Kulturobjekten ist, die im kolonialen Kontext nach Europa gekommen sind. Ihr habt schon angesprochen, dass es auch eine Ausstellung zum Stadtrundgang gab und gibt. Das sind eher traditionelle Formate. Und es ist fraglich, ob sie überhaupt der Sache dienen können.

Ich frage mich auch, ob diese Themen nicht intensiver im Bildungsprogramm integriert sein müssten. Wären nicht auch interaktive Formen der Kooperation denkbar, z. B. dass Künstler_innen aus den Ländern mit deutscher Kolonialgeschichte, wie Tansania oder Namibia, hierherkommen - wenn sie denn Interesse daran haben -, um an den Formaten mitzuarbeiten? Als spekulative Frage: Wie könnte Decolonize Erfurt in fünf Jahren aussehen? 
M.E. Also die Vernetzung findet ja bereits statt. Auch wenn hier in diesem Kontext und in der Arbeit deutschlandweit immer noch die Gefahr besteht, Kolonialgeschichte und deren Gegenwart wieder nur aus der gleichen eurozentrischen Sicht zu bewerten. Immer wieder! Und da ist eine Zusammenarbeit natürlich hundertprozentig sinnvoll. Das Nachwirken der Kolonialgeschichte, die findet ja auch dort in den Ländern statt. Also diese Geschichten verbinden ja, und dann ist es natürlich interessant, diese Verbindungen auch aufrechtzuerhalten, wiederherzustellen, auszugraben und darüber etwas zu hören. Es geht darum, diese andere Perspektive zu hören, wo die Geschichte auch stattgefunden hat. Das Thema erlebbar, spürbar und interaktiver zu machen. Das ist ein ganz spannender Punkt. Denn das meinte ich vorhin auch ein bisschen mit Biografiearbeit. Den Menschen sozusagen auf einer anderen Ebene, nicht auf dieser Sachebene der Kommunikation über sie, zu begegnen.

\section{N.G. Über Personen-Erzählungen meinst du?}

M.E. Ja, vielleicht, gute Narrative zu finden und vielleicht auch Vergleichbares zu suchen, insofern das möglich ist, zur eigenen, persönlichen Erfahrung. Also man könnte dann eben woanders anknüpfen. Das nicht mehr nur rational zu erfassen, sondern intensiver zugänglich zu machen. Verstehen ist ja das eine ...

V.M.W. Klar wäre es großartig, wenn man dieses Programm in alle Bildungspläne und in die Schule reinbringen könnte. Aber das können wir nicht. Die Strukturen sind so rigide, daran haben sich schon so viele Leute die Zähne ausgebissen. Meistens finden solche Themen - bezeichnenderweise - nur über externe Bildungsreferent_innen Eingang in Bildungsinstitutionen. Der Stadtrundgang ist vor allem auch deshalb wichtig, weil er nicht im exklusiven Raum der Uni stattfindet. Und es ist auch wichtig, dass da Leute hinkommen, die vielleicht erwarten, unterhalten zu werden, und die sich dann zwangsläufig mit 
Fragen auseinandersetzen, mit denen sie sich sonst nie beschäftigt hätten. Es ist ein wichtiges Format und es ist ein gutes Format, aber es ist auch nur ein Format. Es muss - um auf Céciles Kommentar zurückzukommen - parallel dazu natürlich weitere Aktivitäten geben. Also wenn man sich z. B. eher für diese erfahrbare Geschichte stark macht, würde das Ganze auch noch mal sehr viel mehr ein Format für ein weißes Publikum sein. Man bräuchte eigentlich, wie es in Köln einmal war, zwei verschiedene Stadtrundgänge. Einen für die PoCGruppen und einen für weiße Gruppen. Das eine ist eher ein EmpowermentAnsatz und das andere ist eher ein Critical-Whiteness-Ansatz.

Ich würde mir für die Decolonize-Gruppen wünschen, dass - weil es eben mehrheitlich weiße Gruppen sind, sowohl hier als auch deutschlandweit - noch viel, viel mehr die Komponente der Unterstützung von migrantischen Gruppen in den Fokus gerückt wird. Dass auch im Zuge der Dekolonisierung Erfurts vermehrt dieser Aspekt der Unterstützung mitgedacht wird, wie es z. B. bei der Debatte um Eisenberg geschehen ist. Hier haben die Erfurter Initiative wie auch Decolonize Jena und das Thüringer Antidiskriminierungsnetzwerk thadine die Kritik der ISD aufgegriffen und sich solidarisiert. Ich würde mir wünschen, dass die Initiative mit dem Namen, den sie sich durch die ganzen Aktivitäten möglicherweise erarbeitet hat, auch Raum und Aufmerksamkeit einräumt für Schwarze Themen. Im Übrigen würde ich mir für jede Gruppe, die im Bereich der Aufarbeitung der Kolonialgeschichte sowie gegen Rassismus arbeitet, wünschen, dass diese zunächst kritisch nach innen guckt. Damit meine ich sowohl die internalisierten Rassismen, die in uns allen schlummern, als auch unsere eigene Positionierung, innerhalb der Gruppe und innerhalb der Gesellschaft.

\section{J.M. Ich würde noch gerne einen weiteren Themenkomplex ins Spiel bringen:} Erfurt hat ja im Gegensatz zu einer Stadt wie Hamburg oder Brüssel, von wo sich beispielsweise das Format des Stadtrundgangs gewissermaßen übernehmen ließe, mit der DDR-Geschichte auch noch eine weitere Schicht in der Stadtgeschichte. Eure Station «Hetze in Erfurt» gibt auch einen Einblick in die Situation z. B. der Vertragsarbeiter_innen in der DDR. Auf eurer Website lässt sich das spannungsreiche Verhältnis zum Rassismus in der DDR nachlesen. ${ }^{13}$ Einerseits gab es infolge von Hetze und tätlichen Übergriffen auf migrantische Arbeiter_innen of Color eine Verfolgung der Täter_innen sowie aufklärerische Maßnahmen von Seiten der Staatssicherheit, um weitere Vorfälle zu verhindern. Andererseits passten derlei Ausschreitungen nicht in das Selbstbild der DDR als anti-imperialistischer Gesellschaft und führten daher nicht zu einer öffentlichen Problematisierung rassistischer Muster. Findet sich etwas von dieser DDR-Geschichte im Heute?

G.S. Bei der Beantwortung der Frage nach den DDR-Verhältnissen und dem andauernden Rassismus lässt sich ein Bogen schlagen zu Fragen der Sichtbarkeit. Und zwar nicht nur von Gegenständen und Themen, sondern auch von Menschen. Es gibt ja Menschen, auch in Thüringen, die dezidiert keine
13 Siehe Luisa Giese, Daniel Langbein, Charlotte Rehling: Hetze in Erfurt: Alltagsrassismen als koloniales Erbe, in: Website Decolonize Erfurt, 15.3.2019, decolonizeerfurt.wordpress.com/hetzein-erfurt/ (19.2.2020). 
14 Siehe Daniel Schulz: «Ostdeutsche sind auch Migranten». Professorin über Identitäten, in: taz, 13.5.2018, taz.de/Professorin-ueberIdentitaeten|!5501987| (18.2.2020).

$15 \mathrm{Vgl}$. z. B. Bonaventure Soh Bejeng Ndikung zur Debatte um das Berliner Schloss und den Palast der Republik: Die gestorben sind, sind niemals fort, Berlin 2018 sowie Thomas Oberender: Die Mauer ist nicht gefallen, in: Die Zeit, 27.9.2017, zeit.de/2017/40/ddr-mauerfallkinderbetreuung-west-ost-deutschland (9.3.2020).

16 Vgl. z. B. Jana Hensel, Wolfgang Engler: Wer wir sind. Die Erfahrung, ostdeutsch zu sein, Berlin 2018, u. a. 53. siehe auch die Ausführungen der Lektorin Maike Nedo: Ich, im Westen undercover, in: Die Zeit, Nr. 29, 12.7.2018, www.zeit.de/2018/29/ostwest-wanderung-gruende-mauerfall (18.2.2020).
Bürger_innen sind. Und die aus dieser Position heraus aktivistisch tätig sind. Ich denke an die Menschen von Refugee Black Box in Jena, die zu gewissen Demos auch an Orte wie Erfurt kommen. Aber das ist eine Position, aus der heraus sie agieren, sprechen, sich Gehör verschaffen können oder eben nicht, die ganz partikular ist. Diese Position zu kontextualisieren und einzubeziehen, ist eine absolute Notwendigkeit und gleichzeitig eine Schwierigkeit. Da geht es dann tatsächlich um die Frage, was eine bestimmte Aktion denn genau für die Menschen bedeutet, bis hin zu ganz existenziellen Fragen. Wenn dann die Polizei kommt, wenn man mit ihnen einen Stadtrundgang macht ... Das sind so Fragen, über die man, glaube ich, noch mal sprechen müsste.

Und um jetzt eben den Bogen zu schlagen: Das sind auch Menschen, die in dem Alltagsbild einer Stadt wie Erfurt nicht präsent sind, weil sie räumlich gesehen marginalisiert sind. Und das ist etwas, was in der DDR natürlich auch den vertragsarbeitenden Menschen angetan wurde. Hier sehe ich eine Verbindung in der partikularen Konstellation von Da-Sein, aber Nicht-sichtbar-Sein, Marginalisiert-Sein, aber trotzdem auch Aktivistisch-tätig-Sein.

N.G. Um an Janas Frage des spezifischen Kontexts der DDR-Geschichte anzuknüpfen, würde ich gerne an dieser Stelle noch die Position der Soziologin Naika Foroutan ins Spiel bringen. ${ }^{14}$ Sie sagt, Menschen aus der DDR und migrierte Menschen haben ähnliche Erfahrungen gemacht, weil sie von der BRD <kolonisiert> wurden bzw. von rassistischen Strukturen in Deutschland betroffen sind. Ich weiß nicht, ob ihr euch da überhaupt positionieren wollt. Ich weiß nämlich auch nicht, ob solche Vergleiche nicht auch extrem problematisch sind, weil sie dazu neigen, eine jahrelange Geschichte von Rassismus als Instrument von weißer Herrschaft zu verdecken und aktuelle Debatten um Rassismus und Migration umzulenken.

U.L. Also die Frage ist ja: Lässt sich das, was nach ' 89 passiert ist mit der DDR, in sinnvoller Weise als Kolonisierung verstehen oder nicht?

N.G. Ich weiß gar nicht, ob Foroutan den Begriff benutzt. Trotzdem kursiert er natürlich als Metapher, z.B. in der Formulierung des innerdeutschen Kolonialismus. ${ }^{15}$ Bei Foroutan geht es eher um eine Argumentation ähnlicher Erfahrungen zwischen Ostdeutschen und Menschen mit Migrationsgeschichte: Gefühle von Heimatverlust, Fremdheitsgefühle und Abwertungen.

J.M. Nicht teilnehmen. Nicht zu den Teilhabenden zu gehören. Die Publizistin Jana Hensel beispielsweise vertritt auch diese Position, zu sagen: Aus der DDR zu kommen, ist auch eine Form der migrantischen Erfahrung. Sie zieht ebenfalls diese Parallele. ${ }^{16}$

M.E. Spannend. Ein bisschen polarisierend, finde ich. Oder? Migration gibt's ja nicht erst seit gestern. Die gehört zu uns dazu, sie gehört zum Menschsein, zur Menschwerdung - oder wie auch immer man das nennen möchte. Sie ist auch 
Teil der Globalgeschichte und speziell für Deutschland könnte man sich dann auch andere Kulturen angucken, z. B. Sudetendeutsche und Schlesier_innen.

C.S. Noch etwas zum Begriff 〈Kolonisierung〉: Also der wurde auch schon an uns herangetragen bei Stadtrundgängen. Da kam auch schon mal jemand zu uns und sagte: «Ach, super, dass Sie sich mit dem Thema beschäftigen.» Und da wurde dann schnell klar, dass die Person geglaubt hatte, wir machen einen Stadtrundgang zur Kolonisierung der DDR.

M.E. In mir brennt noch etwas, ich finde es jetzt auch schwierig in unserer Runde darüber zu sprechen. Nur Jana ist hier sozialisiert, soweit ich das verstanden habe. Wir können ja Jana fragen: Hattest du schon mal ein Gespräch mit einer anderen migrierten Person und hast Vergleiche gezogen? Du kannst auch ein Beispiel nehmen. Ich möchte jetzt nicht, dass du dich persönlich als Beispiel benutzt, wenn du das nicht möchtest.

J.M. Ich kann ja auch nicht für die ehemaligen DDR-Bürger_innen sprechen. Mein Leben in der DDR endete in recht jungen Jahren. Also wenn ich mich mit Personen mit Migrationsbiografie unterhalte, dann habe ich natürlich nicht das Gefühl, dass ich das Gleiche erlebt habe. Das nicht. Weil genau dieses Moment, dass zu den Geschichten von Migrant_innen oft Flucht und Deportation oder eben Verschleppungen gehören und physische Gewalterfahrungen, in meinem Lebenslauf fehlt.

M.E. ... auch sprachliche Barrieren und infolgedessen Ausgrenzung zu erfahren, spielte nach I990 keine Rolle.

J.M. Das stimmt. Eine solche Ausgrenzung aufgrund meiner Sprache habe ich nicht erfahren. Aber es ist interessant, dass ich das jetzt feststelle. Jetzt erst! Also eben $3^{\circ}$ Jahre später habe ich schon den Eindruck, dass tatsächlich bestimmte Formen der Teilhabe für mich nicht zugänglich sind. Das hat u. a. damit zu tun, dass soziale Netzwerke oder Netzwerkformen für uns ... also da gehörten wir nicht rein, da hatten wir keine Anschlüsse, weil wir eben so lange durch eine Grenze von den Netzen abgeschnitten waren. Und tatsächlich gibt es da doch auch ein Sprachproblem, meinem Empfinden nach. Etwa bei Bewerbungsgesprächen - also dann, wenn es darum geht, auf eine gewisse Position zu kommen - fühle ich mich benachteiligt, weil ich in bestimmten Sprechweisen nicht sozialisiert bin und weil ich die Spielregeln nur so halb kenne. Mit dem Soziologen 
17 Vgl. Steffen Mau: Lütten Klein. Leben in der ostdeutschen Transformationsgesellschaft, Berlin 2019, $14 \mathrm{f}$. u. 48. Mau bezieht sich mit dieser Beschreibung auf den Begriff der «arbeiterlichen Gesellschaft» des Soziologen Wolfgang Engler, vgl. ders.: Die Ostdeutschen als Auantgarde, Berlin 2002, insb. 74, $76 \mathrm{f}$.

18 Michael Rothberg: Multidirectional Memory. Remembering the Holocaust in the Age of Decolonization, Stanford 2009.

19 Elsa Koester: Zweihundertneunundfünzigtausend, in: Der Freitag online, 28.10.2019, www.freitag.de/autoren/elsa-koester/ zweihundertneunundfuenzigtausend (18.2.2020).
Steffen Mau z.B. lässt sich das auch als Erbe einer «arbeiterlichen Kultur» fassen, die in der DDR in allen Bereichen vorherrschte. ${ }^{17}$

Mir ist auch geläufig, auf Nicht-verstehen-Wollen zu treffen: also diese Formen der Begegnung mit Westdeutschen - nicht mit allen! -, bei denen spürbar wird, dass es für sie einfach klar ist, dass wir alle nur in der Bundesrepublik Deutschland leben wollen und wollten. Während, glaube ich, es '89, '90 wirklich nicht richtig klar war, was eigentlich die Menschen in der DDR möchten. Also ob sie diese Transition in ein anderes System möchten oder ob es doch nicht auch Überlegungen gab, ein Drittes zu schaffen.

V.M.W. Vielen Dank! Ich finde es gut, dass wir darüber sprechen. Gerade, wenn man in Ostdeutschland decolonize-Arbeit macht, ist dieses Thema wichtig. Ich nehme es als gefährlich wahr, eben weil man dabei auch Dinge gleichsetzt, die überhaupt nicht gleichzusetzen sind, die völlig unterschiedlichen Kontexten entspringen. Also die historische, hierarchische Aufspaltung von Gesellschaften anhand von konstruierten Markern - also z. B. Hautfarbe - wird vermischt mit ganz anderen Erfahrungen zu einer ganz anderen Zeit. Dieses Vermischen ist sehr schade und problematisch und es stellt vor allem eine Konkurrenz her, die überhaupt nicht sein muss. Damit beschäftigen sich Konzepte wie die multidirektionale Erinnerung nach Michael Rothberg. ${ }^{18}$ Es geht darum, verschiedene Erinnerungen gemeinsam zu denken, Verbindungen aufzuzeigen und auch um den grundlegenden Anspruch von Erinnerungskultur, nämlich möglichst respektvoll mit Erfahrenem umzugehen. Da ist gerade in Ostdeutschland viel Potenzial, eben weil die Leute hier Ausgrenzungserfahrungen gemacht haben.

Vielleicht noch ein kleiner Zusatz: Es gibt deshalb auch innerhalb des Decolonize-Netzwerks eine Sektion, in der sich ostdeutsche Decolonize-Gruppen zusammengetan haben, die sich z. B. mit Straßenumbenennungen beschäftigen. Es wurden ja ganz viele Straßen in der DDR umbenannt und danach wieder zurück umbenannt. Spezifisch ostdeutsche Erfahrungen muss man natürlich auch spezifisch angehen.

J.M. Hier lässt sich eine abschließende Frage stellen: Nach der Thüringenwahl hat die Berichterstattung ja umgeschwenkt von «Wir müssen die Ostdeutschen verstehen» oder «Wir müssen die AfD verstehen» auf «Nein, wir müssen uns jetzt endlich eingestehen, dass ein Viertel der Bevölkerung für rechte oder rechtsextreme Positionen einsteht und es total wichtig findet, dass diese im öffentlichen Leben mehr Raum bekommen». Elsa Koester hat in einem Artikel im Freitag deswegen die Frage gestellt, wie wir eigentlich mit rechten Personen, die unsere Kinder betreuen, die unsere Angehörigen pflegen, die in den Gerichten Entscheidungen treffen, mit denen wir in der Bäckerei schwatzen usw. noch reden können: ${ }^{19}$ Wie können wir überhaupt miteinander ins Gespräch kommen? Die Frage an euch wäre: Habt ihr einen Ansatz einer möglichen Antwort darauf, wie man miteinander sprechen lernen kann? 
M.E. Ich sehe das nicht als meine Aufgabe.

C.S. Ich glaube, insgesamt muss man nicht mit allen reden.

M.E. Ich habe meine Position und ich habe meine Erfahrungen hier in diesem Land gemacht, Ost wie West. Sie sind die Grundlage und die Basis für mein Leben und für meine Handlungen. Es ist nicht meine Aufgabe, mit jeder Person in Verbindung zu treten. Sondern im Gegenteil, meine Position zu verteidigen, und zwar nicht nur, weil ich das tun möchte, sondern weil ich das auch tun muss.

U.L. Ich glaube auch, die erste Frage muss sein: Sind diese 25 Prozent für Argumente erreichbar? Das wird man ziemlich schnell merken in einer Kommunikation. Ich denke, wenn sie erreichbar sind, muss man mit ihnen reden, auf jeden Fall. Aber diejenigen, die von vornherein nicht erreichbar sind, die muss man versuchen, konsequent und mit allen durch die Verfassung zur Verfügung stehenden Mitteln zu marginalisieren. Sodass sie im öffentlichen Raum so wenig Präsenz wie möglich haben und ihre rassistischen - und auch sexistischen und ableistischen - Menschenfeindlichkeiten nicht ausleben können. Ich glaube, der Ansatz «Wir müssen mit allen reden und auf alle zugehen» ist falsch.

c.s. Im Übrigen ist dies auch Thema unseres Fünf-Punkte-Programms, das wir heute Abend der Stadt vorlegen werden. ${ }^{20}$ Ein Punkt darin besagt, dass es Aufgabe der Stadt sein soll, zu intervenieren, wenn ein Geschäft sein Schaufenster mit z. B. rassistischen oder antisemitischen Symbolen dekoriert. Auch hier gilt, dass nicht alles sagbar sein kann. Im Übrigen sind wir da ja auch nicht originell in unserer Position. Dazu ist in letzter Zeit viel geschrieben worden.

v.M.W. Ich glaube, mit der Arbeit, die wir machen, wird eigentlich klar, dass wir eine Position vertreten, die offen ist und die erst einmal die Kommunikation sucht. Aber mit allen zu reden, kann nicht das Ziel sein. Erst recht ist das nicht die Aufgabe von Menschen, da schließe ich mich Mirjam an, die beispielsweise aufgrund ihrer äußeren Erscheinung ohnehin in der Schusslinie stehen. Umso wichtiger sind die Arbeit in heterogenen Gruppen und die Kooperationen zwischen Initiativen, die gemeinsam demokratiefördernde und bildungspolitische Arbeit leisten. Ich sehe da aber grundsätzlich die gesamte Gesellschaft in der Verantwortung. Und zwar nicht allein die Zivilgesellschaft, sondern eben vor allem auch die Politik und die Institutionen.
20 Decolonize Erfurt: Wie weiter mit Erfurts kolonialem Erbe?, in: Website von Decolonize Erfurt, 17.11.2019, decolonizeerfurt.wordpress. com/wie-weiter-mit-erfurts-kolonialemerbel (18.2.2020). 